Edgar Jake A. Agullo, MD

Francisco A. Victoria, MD

Department of Otolaryngology -Head and Neck Surgery Ospital ng Maynila Medical Center
Correspondence: Dr. Francisco A. Victoria Department of Otolaryngology -Head and Neck Surgery Ospital ng Maynila Medical Center

Quirino Ave. cor. Roxas Blvd., Malate, Manila 1004 Philippines

Phone: (632) 5246061 local 220

E-mail: ommc_enthns@yahoo.com

Reprints will not be available from the author.

The authors declared that this represents original materia that is not being considered for publication or has not been published or accepted for publication elsewhere, in full or in part, in print or in electronic media; that the manuscript has been read and approved by all the authors, that the requirements for authorship have been met by each author and that each author believes that the manuscript represents honest work.

Disclosures: The authors signed disclosures that there are no financial or other (including personal) relationships, intellectual passion, political or religious beliefs, and institutional affiliations that might lead to a conflict of interest.

\section{Assessment of the Ototoxic Effects of Amikacin and Meropenem among Neonates in a Tertiary Government Hospital}

\begin{abstract}
Objective: Using pre- and post-treatment otoacoustic emission (OAE) tests, this study aimed to assess the ototoxic effect of meropenem, amikacin and meropenem plus amikacin among neonates treated for sepsis neonatorum in a neonatal intensive care unit versus untreated outpatient controls.
\end{abstract}

\section{Methods:}

Design: Prospective Quasi-Experimental Controlled Clinical Trial

Setting: Tertiary Government Hospital

Subjects: Neonates treated for sepsis neonatorum in the Neonatal Intensive Care Unit between August to October 2012 who met inclusion criteria were included in this study. Controls were neonates born in the same institution who were not admitted and did not receive any antibiotic treatment. Excluded were those with APGAR $<5$ at first minute, birth weight $<1000$ grams, clinically evident congenital anomalies and initial "refer" results on OAE.

Neonates were subjected to OAE testing before and after seven days treatment with amikacin, meropenem or a combination of both drugs. Results were analysed using chi-square test. Maternal drug intake, family history of hearing impairment and clinical outcomes (whether expired or discharged improved) were not included in this study. Assessment of ototoxic effects were limited to $\mathrm{OAE}$ alone and not confirmed by $\mathrm{ABR}$.

Results: OAE "refer" rates were as follows: no amikacin and no meropenem, $0 \%(0 / 42) ;$ amikacin only, 33.3\% (3/9); meropenem only, 25\% (2/8) and amikacin and meropenem, 50\% (10/20). Statistical analysis showed that hearing loss was dependent on treatment ( $c 2=23.741, p=<$ 0.001). Overall, statistical analysis showed that there is an increased risk of hearing loss when treated with amikacin and/or meropenem as compared to no treatment.

Conclusion: There is an increased risk of ototoxicity when amikacin, meropenem or a combination of both drugs is administered to neonates. While the ototoxic effects of amikacin have been elucidated, further studies involving meropenem and its potential ototoxic effect are recommended.

Keywords: ototoxicity, amikacin, meropenem, otoacoustic emission testing, neonatal hearing loss 
Ototoxicity refers to the injurious effect of a drug or any chemical substance on the organ of hearing or balance. ${ }^{1}$ Drugs that could inflict such damage include aminoglycoside antibiotics, vancomycin, erythromycin IV, minocycline, amphotericin B, quinine, salicylates, cisplatin and loop diuretics among others. ${ }^{2}$ These drugs can cause auditory and/or vestibular dysfunction. The damage is usually permanent hearing loss and tinnitus secondary to sensorineural degradation. Generally, the site of lesion is almost exclusively cochlear and balance dysfunction may derive from comparable degeneration. ${ }^{3}$ The mechanism of cell damage is mainly through apoptosis particularly on the outer hair cells. ${ }^{4}$

Severe neonatal infections can be treated by various types of antimicrobials. Ideally, culture-guided therapy is recommended to prevent production of multidrug resistant strains. However, empiric therapy is usually initiated in order to facilitate prompt and immediate treatment. As documented by a previous study, ${ }^{5}$ common pathogens in sepsis neonatorum are frequently susceptible to aminoglycosides particularly gentamicin and amikacin as well as to carbapenems such as meropenem and imipenem.

Aminoglycosides are well-known and regarded as a successful class of antibiotics. The first aminoglycoside was streptomycin which was isolated from Streptomyces griseus. This provided treatment for tuberculosis and was found to be effective against gram-negative bacteria. Years after, other aminoglycosides were also isolated from members of the Streptomyces spp. In addition to their potent antimicrobial efficacy, all aminoglycosides can cause toxic side effects on the kidneys and inner ear. While damage inflicted on the kidney is usually temporary, damage to the inner ear is irreversible. ${ }^{6}$

Carbapenems are beta-lactams with the broadest antibacterial spectrum currently available. They are generally well-tolerated and there are only few reports of drug-related adverse events. They have a definite role in empiric and definitive therapy of serious and multi drug resistant bacterial infections. ${ }^{9}$ Interestingly, this group of drugs was developed from a drug called thienamycin which was first detected from a culture of Streptomyces cattleya, a member of the Streptomyces family where aminoglycosides were first isolated from. ${ }^{7}$ The most frequently reported adverse events were diarrhea, rash, nausea and vomiting, thrombocytosis, eosinophilia and changes in hepatic biochemistry. ${ }^{8}$ No data can suggest its possible ototoxic effect.

The American Academy of Audiology released its position statement and policy guidelines in ototoxicity monitoring. ${ }^{3}$ The set of guidelines recommended audiologic monitoring for ototoxicity to be primarily performed for two purposes: "early detection of changes to hearing status presumably attributed to a drug/treatment regime so that changes in the drug regimen may be considered and audiologic intervention when handicapping hearing impairment has occurred." Audiologic monitoring is suggested in cases of treatment using platinum coordination complexes, aminoglycosides, loop diuretics and nonsteroidal anti-inflammatory agents. Audiological methods possibly of value in ototoxicity monitoring include basic audiologic assessment, high frequency audiometry (HFA) and otoacoustic emission (OAE) measurement. These may be used separately or in combination and may vary in utility, reliability and purpose and applicability to specific patient populations. Regardless of the method to be used, it is highly recommended that a baseline evaluation be carried out so that future results will have a good basis of interpretation.

To date, the best approach in evaluating newborn hearing is through otoacoustic emissions (OAE) or auditory brainstem response (ABR) testing. ${ }^{10}$ Otoacoustic emission test equipment is more accessible and mild degrees of motion artifact do not interfere with test results. Moreover, transient-evoked otoacoustic emissions (TEOAE) can be used as a powerful, sensitive and reliable test in evaluation of cochlear damage. ${ }^{14}$ Auditory brainstem response offers a more complete evaluation of the hearing pathway but motion artifacts may affect the test results and if the test cannot be performed because of motion artifacts, sedation may be necessary. ${ }^{11}$

This study aims to assess the ototoxic effect of meropenem, amikacin, and meropenem plus amikacin among admitted neonates treated for sepsis neonatorum in a tertiary government hospital neonatal intensive care unit through pre- and post-treatement otoacoustic emission testing versus outpatient controls. The results of this study may be used in guiding the clinician in administering the involved drugs in order to maximize efficacy and minimize the possible adverse outcomes.

\section{METHODS}

STUDY DESIGN: Prospective Quasi-Experimental Controlled Clinical Trial SETTING: Tertiary Government Hospital

SUBJECTS: Neonates admitted to the neonatal intensive care unit between August to October 2012 for possible sepsis neonatorum and met inclusion criteria were included in this study. The control group was composed of neonates born in the same institution who were not admitted and did not receive any antibiotic treatment.

Neonates with poor APGAR score $<5$ at first minute, extremely low birth weight $<1000$ grams, clinically evident congenital anomalies, and initial "refer" results on OAE were not included to ensure that all subjects were presumed to be of normal hearing function before commencement of the treatment intervention. 


\section{ORIGINAL ARTICLES}

\section{INTERVENTION}

Neonates were systematically assigned to three groups at the time of admission. This was based on the severity of the clinical and laboratory findings as assessed by pediatric residents. Group A neonates (least severe) were given an Aminoglycoside (amikacin at $15 \mathrm{mg} / \mathrm{kg} /$ day), Group B neonates (moderately severe) were given a Carbapenem (meropenem at $20 \mathrm{mg} / \mathrm{kg} /$ dose q12), Group $\mathrm{C}$ neonates (severe) were given combination of amikacin at $15 \mathrm{mg} / \mathrm{kg} /$ day and meropenem at $20 \mathrm{mg} / \mathrm{kg} / \mathrm{dose}$ q12. Informed consent was secured from the parents after informing them the need for admission. Risks and benefits of undergoing the proposed treatment plan were explained to the parents as part of the consent. Otoacaustic emission test using MADSEN AccuScreen ${ }^{\circ}$ PRO v. 1.16E1M (GN Otometrics, Denmark) was performed on all these neonates prior to the start of antibiotics and after seven days of antibiotics and on controls.

Neonates were subjected to OAE testing before and after seven days treatment with amikacin, meropenem or a combination of both drugs. Results were analysed using chi-square test. Maternal drug intake, family history of hearing impairment and clinical outcomes (whether expired or discharged improved) were not included in this study. Assessment of ototoxic effects was limited to OAE alone and not confirmed by ABR.

\section{MAIN AND SECONDARY OUTCOME MEASURE}

A grade of pass or refer in both ears were assigned to each subject after the hearing test.

\section{DATA AND STATISTICAL ANALYSIS}

Data were processed using SPSS v11.5 (SPSS Inc., South Wacker Drive, Chicago, IL 60606-6412) for Windows. Results were analyzed using chi square test. Descriptive statistics were applied on demographic profiles. Cross tabulation of hearing loss before and after treatment on each group was done.

\section{RESULTS}

A total of 79 neonates were included in the study; 47 (59.5\%) were male and 32 (40.5\%) werefemale. Nine neonates ( 9 male, no female) were in Group A (least severe, amikacin group), 8 neonates ( 4 male, 4 female) were in Group B (moderately severe, meropenem group), 20 neonates (6 male, 14 female) were in Group C (severe, amikacin + meropenem group) and 42 (28 male, 14 female) were outpatient controls. For the overall age of gestation based on Ballard's Score, 42 neonates (53\%) belonged to the age group of 37-39 weeks. Seventeen (17) of 37 neonates (46\%) from the treatment group had ages of gestation of 3436 weeks while most $(74 \%)$ of the babies from the control group had a Ballards score of 37-39 weeks. (Figure 2) The birth weight among the subjects was also noted and grouped (Figure 2) where 14 neonates (37.8\%) from the treatment group had normal birthweight $(2.5 \mathrm{~kg}-$ $3.0 \mathrm{~kg}$ ) and 18 neonates (48.6\%) had low birthweight. Thirty one (31) neonates (73.8\%) from the control group had normal birthweight.

Baseline OAE results of all subjects were "pass." Hearing tests were repeated after treatment with antibiotic. (Table 1) OAE "refer" rates were as follows: no amikacin and no meropenem, $0 \%$ (0/42); amikacin only, $33.3 \%(3 / 9)$; meropenem only, 25\% (2/8) and amikacin and meropenem, $50 \%(10 / 20)$. Statistical analysis showed that risk of developing hearing loss is dependent on treatment (c2 $=23.741, \mathrm{p}=<0.001$ ). (Table 2) The Chi-square test revealed that there was a significant difference between the proportion of neonates who developed hearing loss after treatment compared with the control group.

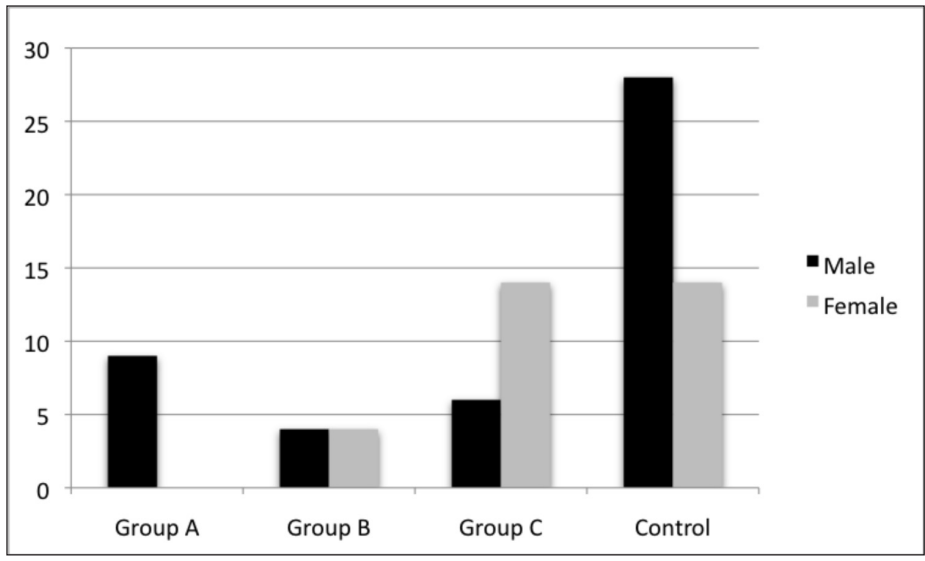

Figure 1. Distribution of Neonates based on Sex between Group A (amikacin), B (meropenem) $C$ (meropenem and amikacin) and Control Group (No Treatment)

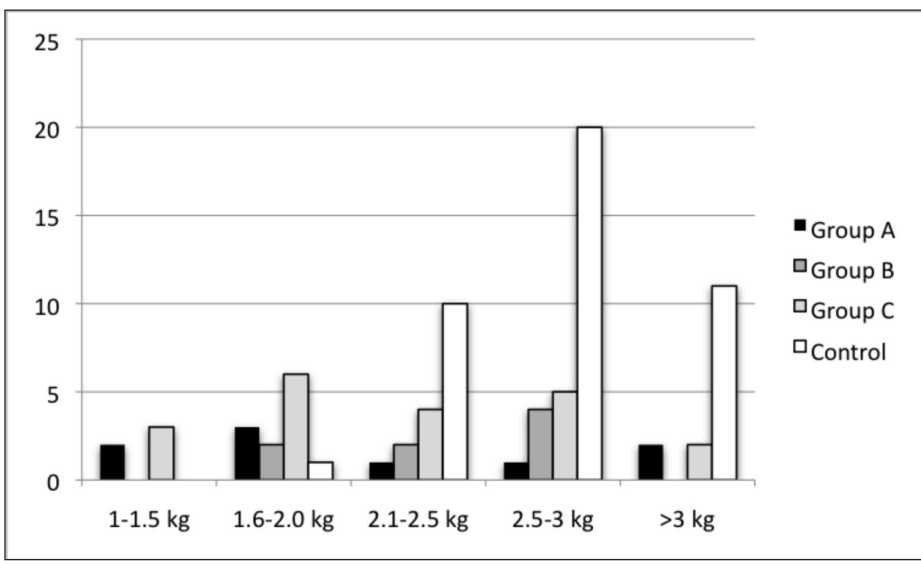

Figure 2. Distribution of Neonates based on Birthweight between Group A (amikacin), B (meropenem), $\mathrm{C}$ (meropenem and amikacin) and Control Group (No Treatment) 
ORIGINAL ARTICLES

Overall, statistical analysis showed that there was an increased risk of developing hearing loss as suggested by a "refer" OAE result when treated with amikacin and/or meropenem as compared to no treatment ( $c^{2}=1.242, p=0.265$ ). (Table 3 ) There was an increased risk of developing hearing loss when treated with amikacin (Group A) as compared to no treatment. For Group B, there was an increased risk of having hearing loss when treated with meropenem as compared to no treatment. For Group C, there was an increased risk of having hearing loss when treated with both meropenem and amikacin as compared to no treatment.

Table 1. OAE results after Treatment

\begin{tabular}{|l|l|c|c|c|}
\hline \multicolumn{2}{|c|}{} & \multicolumn{2}{|c|}{ Tragal pointer } & \multirow{2}{*}{ Total } \\
\cline { 3 - 4 } \multicolumn{2}{c|}{ Treatment } & "Pass" & "Refer" & \\
\cline { 2 - 4 } & No treatment & 42 & 0 & 42 \\
\cline { 2 - 5 } & meropenem & 6 & 2 & 8 \\
\cline { 2 - 4 } & amikacin & 6 & 3 & 9 \\
\cline { 2 - 4 } & $\begin{array}{l}\text { amikacin }+ \\
\text { meropenem }\end{array}$ & 10 & 10 & 20 \\
\hline Total & 64 & 15 & 79 \\
\hline
\end{tabular}

Table 2. Computed Relative Risks Using Chi-square Test among the Treatment Groups

\begin{tabular}{|c|c|c|c|}
\hline \multirow[t]{6}{*}{ meropenem } & Chi-squared & $=5.419$ & p-value $=0.02$ \\
\hline & & Estimate & $95 \% \mathrm{Cl}$ \\
\hline & RRR & -22.889 & {$[-455.485$ to -0.25$]$} \\
\hline & ARR & -0.266 & {$[-0.587$ to -0.058$]$} \\
\hline & NNT & -4 & {$[-2$ to -17$]$} \\
\hline & $\mathbf{R R}$ & 23.889 & [456.485 to 1.25$]$ \\
\hline \multirow[t]{6}{*}{ amikacin } & Chi-squared & $=8.905$ & $p$-value $=0.003$ \\
\hline & & Estimate & $95 \% \mathrm{Cl}$ \\
\hline & RRR & -29.1 & {$[-536.383$ to -0.686$]$} \\
\hline & ARR & -0.338 & {$[-0.635$ to -0.107$]$} \\
\hline & NNT & -3 & [-2 to -9$]$ \\
\hline & $\mathbf{R R}$ & 30.1 & [537.383 to 1.686$]$ \\
\hline \multirow{6}{*}{$\begin{array}{l}\text { meropenem } \\
\text { and amikacin }\end{array}$} & Chi-squared & $=20.336$ & $p$-value $<0.001$ \\
\hline & & Estimate & $95 \% \mathrm{Cl}$ \\
\hline & RRR & -42 & {$[-698.132$ to -1.645$]$} \\
\hline & ARR & -0.488 & {$[-0.685$ to -0.272$]$} \\
\hline & NNT & -3 & {$[-1$ to -4$]$} \\
\hline & $\mathbf{R R}$ & 43 & [699.132 to 2.645$]$ \\
\hline
\end{tabular}

Table 3. Computed Relative Risks Using Chi-square Test among Treatment versus Nontreatment Groups

\begin{tabular}{|l|l|l|l|}
\hline \hline Treatment vs & Chi-squared & $=17.562$ & $\mathrm{p}$-value $<0.001$ \\
\cline { 2 - 4 } No Treatment & & Estimate & $\mathbf{9 5 \%} \mathbf{C l}$ \\
\cline { 2 - 4 } & $\mathbf{R R R}$ & -34.079 & {$[-565.652$ to -1.172$]$} \\
\cline { 2 - 4 } & $\mathbf{A R R}$ & -0.396 & {$[-0.554$ to -0.229$]$} \\
\cline { 2 - 4 } & $\mathbf{N N T}$ & -3 & {$[-2$ to -4$]$} \\
\cline { 2 - 4 } & $\mathbf{R R}$ & 134.079 & {$[665.652$ to 101.172$]$} \\
\hline
\end{tabular}

\section{DISCUSSION}

The ototoxic effects of amikacin have long been proven. Among the aminoglycosides, amikacin has been found to have higher rates of cochleotoxicity. ${ }^{12}$ Previous studies documented the toxic effects of amikacin on high frequencies particularly on levels of $8,000 \mathrm{~Hz}, 4,000$ $\mathrm{Hz}$ and even $2,000 \mathrm{~Hz} \cdot{ }^{13}$ As such, careful monitoring of ototoxicity is recommended whenever the drug is used. Concentration of the drug is initially highest at the basal level of the cochlea thus exerting its effect in this region. ${ }^{14}$ Within this area, outer hair cells are usually the ones that receive the harmful effect. Damage to the outer hair cells can be documented through otoacoustic emissions testing. The OAE device utilized in this study analyzed frequencies of $1500 \mathrm{~Hz}, 2000 \mathrm{~Hz}, 3000$ $\mathrm{Hz}$ and $4000 \mathrm{~Hz} .^{15}$

Meropenem, as a member of the class of drugs called carbapenems, can induce CNS toxicity usually presented as seizures. ${ }^{16}$ Meropenem levels have been quantified in different tissues and body fluids such as CSF, respiratory tract, urinary tract and even in gynecologic tissues but no reports have been made regarding its presence in the inner ear. ${ }^{17}$ Furthermore, no reports have been established for its possible effect on the vestibulocochlear system. Owing to the fact that carbapenems and aminoglycosides have the same origin on Streptomyces spp., ${ }^{6,7}$ we assumed that there may be some similarities in their pharmacokinetics. One possible hint is the nephrotoxicity which is a common characteristic of these groups of drugs. $., 22,24$

In this study, monotherapy with both amikacin and meropenem showed an increased risk of developing hearing loss after seven days of treatment. This is expected of amikacin but not of meropenem. No available literature has been found to explain the possible mechanism of ototoxicity brought about by meropenem. Amikacin stimulates generation of reactive oxygen species (ROS) in the inner ear. Reactive oxygen species can activate cell-death pathways thereby causing irreversible injury to the outer hair cell that could ultimately lead to hearing loss. ${ }^{4}$ On the other hand, meropenem's structure, believed to be the factor responsible for its epileptogenic activity, has not been probed for its possible ototoxic effects. 
Monotherapy with amikacin and combination therapy with amikacin plus meropenem carries the same risk of developing hearing loss. Following this, it may be hypothesized that there is possibly no synergistic effect on ototoxicity of the two drugs.

This study suggested the possible ototoxic effect of meropenem and confirmed that of amikacin on neonates. However, the specific area of the inner ear as predicted by the frequency of hearing impairment where meropenem might have been taking its effect was not established due to the limitation of equipment. It is recommended to test for possible ototoxic effects of meropenem on a larger sample size using equipment that could determine the specific frequency of possible area of injury. Further biochemical studies can also be recommended for meropenem in order to verify the possible mechanism of injury it can induce in the inner ear.

\section{REFERENCES}

1. World Health Organization: Programme for the Prevention of Deafness and Hearing Impairment Report of an Informal Consultation on Strategies for Prevention of Hearing Impairment from Otottoxic Drugs. Geneva, 1994 Nov 21-23.

2. Fairbanks D. Pocket Guide to Antimicrobial Therapy in Otolaryngology - Head and Neck Surgery. 13th ed. American Academy of Otolaryngology--Head \& Neck Surgery Foundation Inc. One Prince Street Alexandria, VA 22314-3357, U.S.A. 2007.

3. Durrant J, Campbell K, Fausti S, Guthrie O, Jacobson G, Lonsbury-Martin B, Poling G. American Academy of Audiology Position Statement and Clinical Practice Guidelines: Ototoxicity Monitoring. American Academy of Audiology. 2009 Oct.

4. Tabuchi K, Nishimura B, Nakamagoe M, Hayashi K, Nakayama M,Hara A. Ototoxicity:mechanisms of cochlear impairment and its prevention. Curr Med Chem. 2011; 18(31):4866-71.

5. Querimit EM. "Outcome of Neonates with Burkholderia cepacia Growth on Blood Culture in a Tertiary Government Hospital from 2009-2011". Presented at Ospital ng Maynila Department of Pediatrics Descriptive Research Presentation, 2012. (Unpublished)

6. Huth ME, Ricci AJ, Cheng AG. Mechanisms of aminoglycoside ototoxicity and targets of hair cell protection. Int J Otolaryngol. 2011; 2011: 937861.

7. Shah PM. Parenteral carbapenems. Clin Microbiol Infect. 2008 Jan; 14 Suppl 1: 175-180.

8. Norrby SR, Newell PA, Faulkner KL, Lesky W. Safety profile of meropenem: international clinical experience based on the first 3125 patients treated with meropenem. J Antimicrob Chemother. 1995 Jul; 36 Suppl A: 207-223.

9. Baldwin CM, Lyseng-Williamson KA, Keam SJ. Meropenem : a review of its use in the treatment of serious bacterial infections. Drugs. 2008; 68(6): 803-38.

10. World Health Organization. Newborn and infant hearing screening: current issues and guiding principles for action. World Health Organization 2010. Geneva 27, Switzerland.

11. Harlor AD Jr, Bower C. Hearing assessment in infants and children: recommendations beyond neonatal screening. Pediatrics. 2009 Oct; 124(4):1252-63. Epub 2009 Sep 28.

12. Javadi MR, Abtahi B, Gholamia K, Safari Moghadam B, Tabarsi P, Salamzadeh J. The incidence of Amikacin Ototoxicity in Multidrug-Resistant Tuberculosis Patients. Iran J Pharm Res. 2011 Fall; 10 (4): 905-911.

13. Black RE, Lau WK, Weinstein RJ, Young LS, Hewitt WL. Ototoxicity of amikacin. Antimicrob Agents Chemother. 1976 Jun; 9(6): 956-961.

14. Naemi M, Maamouri G, Boskabadi H. Assessment of aminoglycoside-induced hearing impairment in hospitalized neonates by TEOAE. Indian J Otolaryngol Head Neck Surg. 2009 Dec 61 (4):256-261.

15. MADSEN AccuScreen PRO Datasheet. Product Insert. Fischer-Zoth Diagnosesysteme GmbH Walter-Kolbenhoff-St. 34 D - 82110 Germering

16. Papp-Wallace KM, Endimiani A, Taracila MA, Bonomo RA. Carbapenems: past, present, and future. Antimicrob Agents Chemother. 2011 Nov; 55(11): 4943-4960.

17. Craig WA. The pharmacology of meropenem, a new carbapenem antibiotic. Clin Infect Dis. 1997 Feb; 24 Suppl 2: S266-75 